\title{
GSC Diversity Receivers over Generalized-Gamma Fading Channels
}

\author{
Petros S. Bithas, Student Member, IEEE, Nikos C. Sagias, Member, IEEE, \\ and P. Takis Mathiopoulos, Senior Member, IEEE
}

\begin{abstract}
A detailed performance analysis of generalizedselection combining $\operatorname{GSC}(2, L)$ receivers operating over generalized-Gamma fading channels is presented. For this class of receivers, a novel closed-form expression for the moments output signal-to-noise ratio is derived. Furthermore, infinite series representations for the moments-generating and the cumulative distribution functions are obtained. The proposed mathematical analysis is accompanied by various performance evaluation results. These theoretical results are complemented by equivalent computer simulated results, which validate the accuracy of the proposed analysis.
\end{abstract}

Index Terms-Generalized-selection combining (GSC), diversity, generalized Gamma distribution, bit error rate (BER).

\section{INTRODUCTION}

$\mathbf{G}$ ENERALIZED-selection combining (GSC) is considered as an alternative diversity scheme for bridging the performance gap between the two classical diversity schemes namely, maximal ratio combining (MRC) and selection combining (SC) [1]. More specifically, as compared to MRC, which is an optimal combining scheme, GSC has reduced system complexity, while as compared to SC, which is one of the simplest diversity schemes, it has improved performance. In the past the performance of GSC diversity receivers has been analyzed for various fading models, including Rayleigh, Nakagami- $m$, and Weibull, e.g., [1]-[3]. Furthermore, special attention is given to the practical important class of $\operatorname{GSC}(2, L)$, e.g., [4], [5], where among $L$ available resolvable paths the two strongest are adaptively combined. Since this class of receivers is an effective compromise achieving very good performance with reduced implementation complexity, it is considered also here in the context of generalized-Gamma (GG) fading channels.

The GG distribution is a generic and versatile fading channel model since it not only includes the Rayleigh, Nakagami$m$, and Weibull distributions as special cases but also can describe the lognormal as a limiting case. Furthermore, it is considered to be mathematically tractable, as compared to various lognormal-based models, and recently has gained increased interest in the field of digital communications over fading channels [6]-[8]. For example, in [6] the outage probability (OP) of dual-branch SC was obtained, while in [7] the

Manuscript received July 3, 2007. The associate editor coordinating the review of this letter and approving it for publication was Prof. Hsiao-Hwa Chen.

P. S. Bithas and P. T. Mathiopoulos are with the Institute for Space Applications and Remote Sensing, National Observatory of Athens, Metaxa \& Vas. Pavlou Street, Palea Penteli, 15236 Athens, Greece (e-mail: \{pbithas, mathio\}@space.noa.gr).

N. C. Sagias is with the Institute of Informatics and Telecommunications, National Centre for Scientific Research-"Demokritos," Agia Paraskevi, 15310 Athens, Greece (e-mail: nsagias@ieee.org).

Digital Object Identifier 10.1109/LCOMM.2007.071095. performance analysis of switch and stay combining diversity receivers was studied. However, the performance of $\operatorname{GSC}(2, L)$ receivers over GG fading channels has not been yet studied in the open technical literature and thus is the subject of this letter.

\section{THE GG FADING ModeL}

Let us consider a diversity receiver operating over $L$ identically distributed (i.d.) GG flat fading channels. The probability density function (PDF) of the instantaneous signal-to-noise ratio (SNR) per symbol at the $\ell$ th input branch $(\ell=1,2, \ldots, L)$ is $[7$, eq. (1)]

$$
f_{\gamma_{\ell}}(\gamma)=\frac{\beta \gamma^{m \beta / 2-1}}{2(m-1) !(\tau \bar{\gamma})^{m \beta / 2}} \exp \left[-\left(\frac{\gamma}{\tau \bar{\gamma}}\right)^{\beta / 2}\right]
$$

where $\beta>0$ and $m=1,2, \ldots$ are two parameters related to the fading severity, $\bar{\gamma}$ is the average input SNR per symbol, and $\tau=(m-1) ! / \Gamma(m+2 / \beta)$, with $\Gamma(\cdot)$ being the Gamma function [9, eq. (8.310/1)]. By using different values of $\beta$ and $m$, (1) can describe a great variety of short- and long-term (shadowing) fading conditions [7]. Moreover, the cumulative distribution function (CDF) of $\gamma_{\ell}$ can be expressed as

$$
F_{\gamma_{\ell}}(\gamma)=1-\exp \left[-\left(\frac{\gamma}{\tau \bar{\gamma}}\right)^{\beta / 2}\right] \sum_{i=0}^{m-1} \frac{1}{i !}\left(\frac{\gamma}{\tau \bar{\gamma}}\right)^{i \beta / 2}
$$

while the $n$th order moment of $\gamma_{\ell}$ is given by

$$
\mu_{\gamma_{\ell}}(n)=(\tau \bar{\gamma})^{n} \frac{\Gamma(m+2 n / \beta)}{(m-1) !} .
$$

\section{GSC $(2, L)$ ReCEIVER SNR OUtPut Statistics}

Let us order the $\gamma_{\ell}$ 's as $\gamma_{(1)} \geq \gamma_{(2)} \geq \cdots \geq \gamma_{(L)}$. The instantaneous SNR per symbol at the output of a $\operatorname{GSC}(2, L)$ receiver is $\gamma_{\mathrm{gsc}}=\gamma_{(1)}+\gamma_{(2)}$. The joint PDF of this ordered set can be mathematical expressed as [2, eq. (15)]

$$
f_{\gamma_{(1)}, \gamma_{(2)}}\left(\gamma_{1}, \gamma_{2}\right)=\frac{L !}{(L-2) !} f_{\gamma_{1}}\left(\gamma_{1}\right) f_{\gamma_{2}}\left(\gamma_{2}\right)\left[F_{\gamma_{2}}\left(\gamma_{2}\right)\right]^{L-2}
$$

Starting with (2), using first the binomial theorem [9, eq. (1.111)], then the multinomial identity [10, eq. (24.1.2)], and after some mathematical simplifications, $\left[F_{\gamma_{2}}\left(\gamma_{2}\right)\right]^{L-2}$ in (4) can be expressed as

$$
\begin{aligned}
& {\left[F_{\gamma_{2}}\left(\gamma_{2}\right)\right]^{L-2}=\sum_{k=0}^{L-2} \sum_{\substack{n_{0}, n_{1}, \ldots, n_{m-1}=0 \\
n_{0}+n_{1}+\cdots+n_{m-1}=k}}^{k} \frac{k !\left(\begin{array}{c}
L-2 \\
k
\end{array}\right)(-1)^{k}}{\prod_{i=0}^{m-1} n_{i} !(i !)^{n_{i}}}} \\
& \times\left(\frac{\gamma_{2}}{\tau \bar{\gamma}}\right)^{\sum_{i=0}^{m-1} i n_{i} \beta / 2} \exp \left[-k\left(\frac{\gamma_{2}}{\tau \bar{\gamma}}\right)^{\beta / 2}\right] .
\end{aligned}
$$




\section{A. Moments}

The $n$th order moment of a GSC diversity receiver output SNR is given by $\mu_{\text {gsc }}(n)=\mathbb{E}\left\langle\gamma_{\text {gsc }}^{n}\right\rangle$, with $\mathbb{E}\langle\cdot\rangle$ denoting expectation. Using the binomial theorem [9, eq. (1.111)], it can be written as

$$
\mu_{\mathrm{gsc}}(n)=\sum_{p=0}^{n}\left(\begin{array}{l}
n \\
p
\end{array}\right) \mathbb{E}\left\langle\gamma_{(1)}^{p} \gamma_{(2)}^{n-p}\right\rangle
$$

Hence, by substituting (1), (4) and (5) in (6), and after making a change of variables, integrals of the following form need to be solved $\mathcal{I}=$ $\int_{0}^{\infty} \gamma_{1}^{c} \exp \left(-C \gamma_{1}\right) \int_{0}^{\gamma_{1}} \gamma_{2}^{d} \exp \left(-D \gamma_{2}\right) \mathrm{d} \gamma_{2} \mathrm{~d} \gamma_{1}$, with $C$, $D$ being positive integers. Using [9, eqs. (3.381/1) and $(6.455 / 2)], \mathcal{I}$ can be solved in closed form and consequently, the moments of the GSC output SNR can be expressed as

$$
\begin{aligned}
& \mu_{\mathrm{gsc}}(n)=L ! \frac{(\tau \bar{\gamma})^{n}}{[(m-1) !]^{2}} \sum_{p=0}^{n}\left(\begin{array}{c}
n \\
p
\end{array}\right) \\
& \times \sum_{k=0}^{L-2} \sum_{\substack{n_{0}, n_{1}, \ldots, n_{m-1}=0 \\
n_{0}+n_{1}+\cdots+n_{m-1}=k}}^{k} \frac{(-1)^{k} \Gamma[\mathcal{G}(2, n)]}{(k+2)^{\mathcal{G}(2, n)}(L-2-k) !} \\
& \times \frac{{ }_{2} F_{1}\left[1, \mathcal{G}(2, n) ; 1+\mathcal{G}(2, n-p-m \beta / 2) ; \frac{k+1}{k+2}\right]}{\mathcal{G}(2, n-p-m \beta / 2) \prod_{i=0}^{m-1} n_{i} !(i !)^{n_{i}}}
\end{aligned}
$$

where $\mathcal{G}(a, b)=a\left[(m+b / \beta)+\sum_{i=0}^{m-1} i n_{i} / 2\right]$ and ${ }_{2} F_{1}(\cdot, \cdot ; \cdot ; \cdot)$ is the Gauss hypergeometric function [9, eq. (9.100)]. It should be noted that by setting $m=n=1$ in (7), the expression for $\mu_{\mathrm{gsc}}$ simplifies to a previously known result [3, eq. (8)], i.e., the average output SNR of a GSC receiver operating over Weibull fading channels.

For the special case of MRC, by considering not necessarily i.d. GG fading conditions, i.e., each channel is characterized by different fading parameters $m_{\ell} \geq 1 / 2, \beta_{\ell}>0$ and average input SNR per symbol $\bar{\gamma}_{\ell}$, a general result can be obtained. By substituting the output of the MRC receiver, $\gamma_{\mathrm{mrc}}=\sum_{\ell=1}^{L} \gamma_{\ell}$, in $\mu_{\mathrm{mrc}}(n)=\mathbb{E}\left\langle\gamma_{\mathrm{mrc}}^{n}\right\rangle$, using (3) and the multinomial identity, the moments of the MRC output SNR can be expressed as

$$
\mu_{\mathrm{mrc}}(n)=n ! \sum_{\substack{n_{0}, n_{1}, \ldots, n_{L}=0 \\ n_{0}+n_{1}+\cdots+n_{L}=n}}^{n} \prod_{\ell=1}^{L}\left(\tau_{\ell} \bar{\gamma}_{\ell}\right)^{n} \frac{\Gamma\left(m_{\ell}+2 n / \beta_{\ell}\right)}{n_{\ell} ! \Gamma\left(m_{\ell}\right) !}
$$

with $\tau_{\ell}=\Gamma\left(m_{\ell}\right) / \Gamma\left(m_{\ell}+2 / \beta_{\ell}\right)$.

\section{B. Moments-Generating Function (MGF)}

Since the MGF of $\gamma_{\mathrm{gsc}}$ is given by $\mathbb{M}_{\gamma_{\mathrm{gsc}}}(s)=$ $\mathbb{E}\left\langle\exp \left[-s\left(\gamma_{(1)}+\gamma_{(2)}\right)\right]\right\rangle$, substituting (1), (4), and (5) in $\mathbb{M}_{\gamma_{\mathrm{gsc}}}(s)$ and using an infinite series representation for the exponential functions [9, eq. (1.211/1)], similar integrals to $\mathcal{I}$ appear. Hence, using [9, eqs. (3.381/1) and (6.455/2)], $\mathbb{M}_{\gamma_{\mathrm{gsc}}}(s)$ can be obtained in terms of an infinite series repre- sentation as

$$
\begin{aligned}
& \mathbb{M}_{\gamma_{\mathrm{gsc}}}(s)=\frac{\beta L !}{[(m-1) !]^{2}} \sum_{k=0}^{L-2} \sum_{\substack{n_{0}, n_{1}, \ldots, n_{m-1}=0 \\
n_{0}+n_{1}+\cdots+n_{m-1}=k}}^{k} \sum_{t, h=0}^{\infty} \\
& \times \frac{(-1)^{k+t+h}(k+1)^{t} \Gamma[\mathcal{G}(\beta, \beta(t+h) / 2)]}{t ! h !(L-2-k) ! \prod_{i=0}^{m-1} n_{i} !(i !)^{n_{i}}} \\
& \times \frac{{ }_{2} F_{1}[1, \mathcal{G}(\beta, \beta(h+t) / 2) ; 1+\mathcal{G}(\beta, \beta(t-m) / 2) ; 1 / 2]}{(2 s \tau \bar{\gamma})^{\mathcal{G}(\beta, \beta(t+h) / 2)} \mathcal{G}(2, \beta(t-m) / 2)} .
\end{aligned}
$$

\section{Cumulative Distribution Function $(C D F)$}

Applying the inverse Laplace transform in (9), i.e., $\mathscr{L}^{-1}\left[\mathbb{M}_{\gamma_{\text {gsc }}}(s) / s ; \gamma\right]$, and after some straight-forward mathematical simplifications, the CDF of $\gamma_{\mathrm{gsc}}$ can be obtained as

$$
\begin{aligned}
& \mathbb{F}_{\gamma_{\mathrm{gsc}}}(\gamma)=\frac{L !}{[(m-1) !]^{2}} \sum_{k=0}^{L-2} \sum_{\begin{array}{c}
n_{0}, n_{1}, \ldots, n_{m-1}=0 \\
n_{0}+n_{1}+\cdots+n_{m-1}=k
\end{array}}^{k} \sum_{t, h=0}^{\infty} \\
& \times \frac{(-1)^{k+t+h}(k+1)^{t} \gamma^{\mathcal{G}(\beta, \beta(t+h) / 2)}}{t ! h !(L-2-k) !(2 \tau \bar{\gamma})^{\mathcal{G}(\beta, \beta(t+h) / 2)} \prod_{i=0}^{m-1} n_{i} !(i !)^{n_{i}}} \\
& \times \frac{{ }_{2} F_{1}[1, \mathcal{G}(\beta, \beta(h+t) / 2) ; 1+\mathcal{G}(\beta, \beta(t-m) / 2) ; 1 / 2]}{\mathcal{G}(2, \beta(t-m) / 2) \mathcal{G}(2, \beta(t+h) / 2)} .
\end{aligned}
$$

\section{PERformance EVAluation AND Discussion}

\section{A. Amount of Fading $(A o F)$}

The AoF, can be expressed in terms of the first- and secondorder moments as $A_{F}=\mu_{\text {gsc }}(2) / \mu_{\text {gsc }}^{2}(1)-1$. In Fig. 1, AoF is plotted as a function of $\beta$, for $\operatorname{GSC}(2,3)$ and $\operatorname{MRC}(L=3)$ and for several values of $m$. As illustrated, for $m=1$ the AoF of GSC and MRC receivers is almost identical, while for higher values of $m$ small performance differences are observed.

\section{B. Average Bit Error Probability (ABEP)}

Using (9) and following the MGF-based approach [1] the ABEP is evaluated. More specifically the ABEP has been obtained and is plotted in Fig. 2 as a function of the average input SNR per bit, $\bar{\gamma}_{\mathrm{b}}=\bar{\gamma} / \log _{2}(M)$, with $M$ being the modulation order. Gray-encoded $M$-ary Phase Shift Keying (PSK), Binary PSK (BPSK), and Differential BPSK (DBPSK) are assumed, for $\operatorname{GSC}(2,3)$ and $\operatorname{GSC}(2,4)$, while $m=2$ and $\beta=3$. As expected, in all cases, $\operatorname{GSC}(2,4)$ outperforms $\operatorname{GSC}(2,3)$. It should be noted that the infinite series expressions of the MGF converge rapidly, especially for increased values of the average input SNR. It is also depicted in Fig. 2 that the theoretical results are in excellent agreement with the equivalent performance evaluation results obtained by means of computer simulations, thus verifying the analysis presented in Section III. 


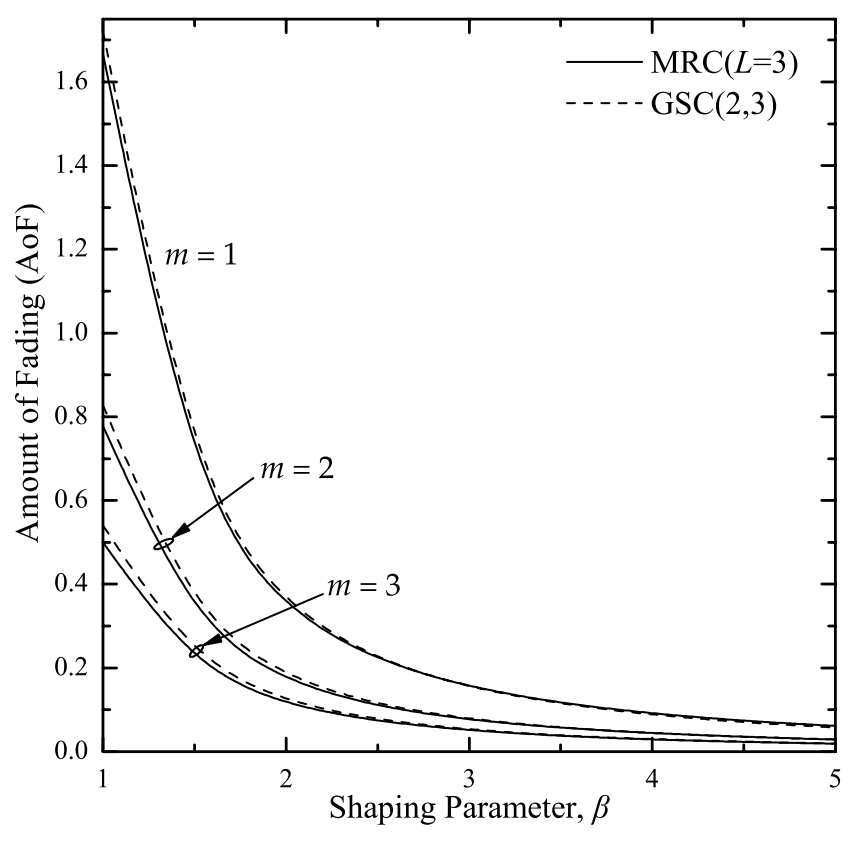

Fig. 1. AoF vs. shaping parameter $\beta$, for several values of $m$ and for $\operatorname{GSC}(2,3)$ and $\operatorname{MRC}(L=3)$.

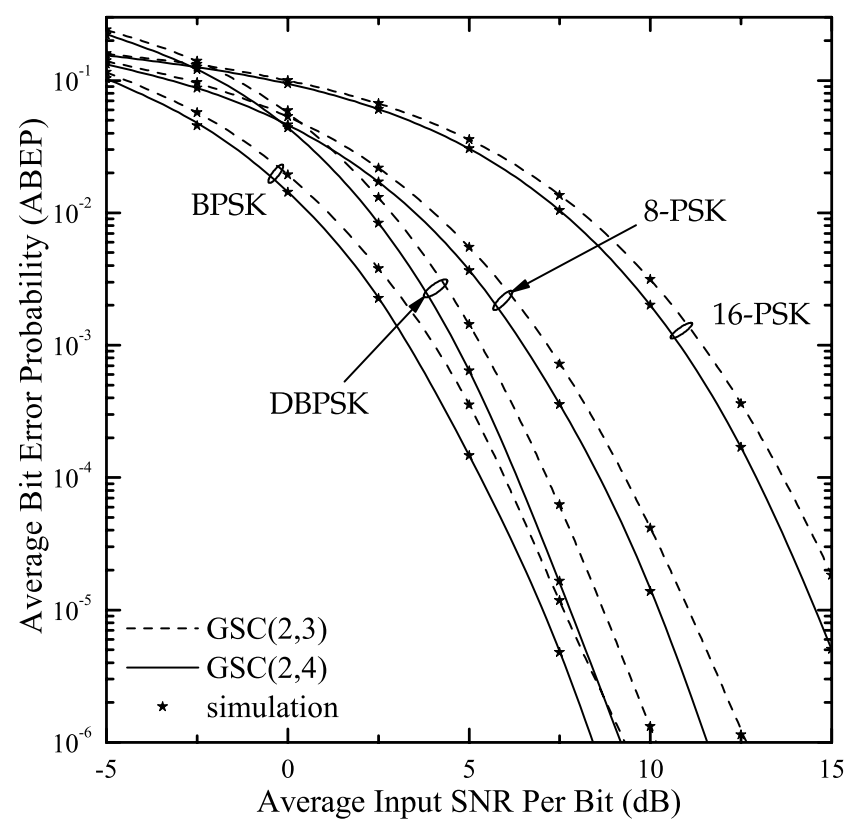

Fig. 2. ABEP of DBPSK, BPSK, Gray-encoded 8-PSK and 16-PSK signaling vs. $\bar{\gamma}_{b}$, for $\operatorname{GSC}(2,3)$ and $\operatorname{GSC}(2,4)$.

\section{Outage Probability $(O P)$}

The OP is defined as the probability that the GSC output SNR falls below a given outage threshold $\gamma_{\text {th }}$ and can be obtained as $P_{\text {out }}\left(\gamma_{\text {th }}\right)=\mathbb{F}_{\gamma_{\text {gsc }}}\left(\gamma_{\text {th }}\right)$. Using (10), $P_{\text {out }}\left(\gamma_{\text {th }}\right)$ is plotted in Fig. 3 as a function of the normalized outage threshold, $\gamma_{\mathrm{th}} / \bar{\gamma}$, for $\operatorname{GSC}(2,3)$ and $\operatorname{GSC}(2,4)$, different values of $m$ and $\beta=3$. It is depicted that for low values of $\gamma_{\text {th }} / \bar{\gamma}$, $\operatorname{GSC}(2,4)$ for $m=2$ has almost the same performance as $\operatorname{GSC}(2,3)$ for $m=3$. Note again the excellent agreement between the theoretical and computer simulation results.

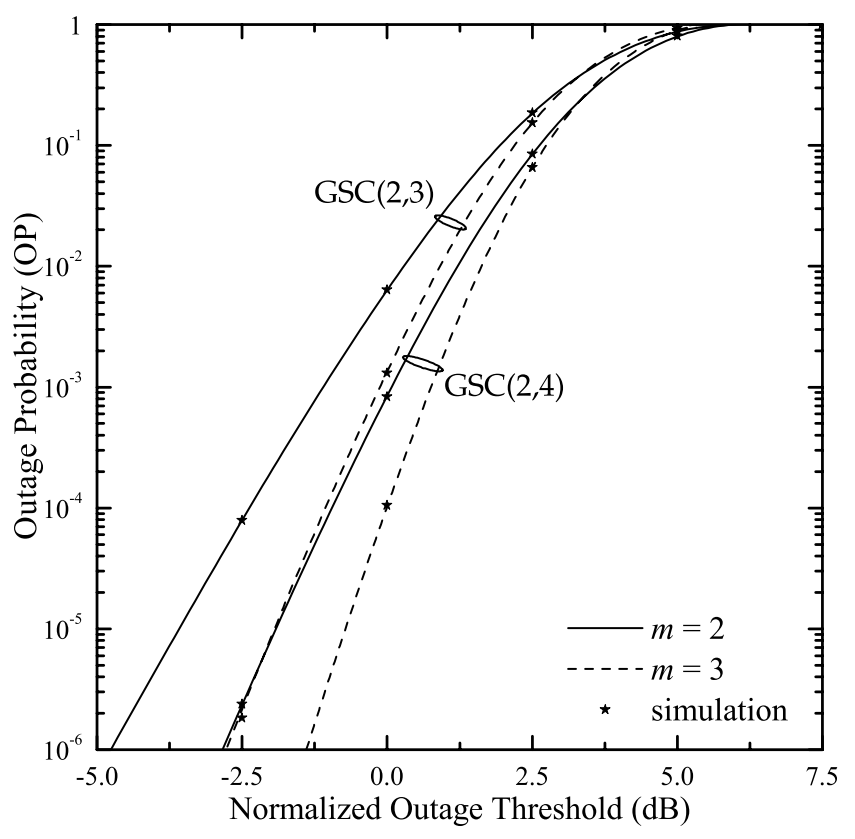

Fig. 3. $P_{\text {out }}$ vs. normalized outage threshold, for $\operatorname{GSC}(2,3)$ and $\operatorname{GSC}(2,4)$ and several values of $m$ and $\beta$.

\section{ACKNOWLEDGMENTS}

This work has been performed within the framework of the SatNEx-II project (IST-027393) funded by European Commission under the FP6 program.

\section{REFERENCES}

[1] M.-S. Alouini and M. K. Simon, "An MGF-based performance analysis of generalized selection combining over Rayleigh fading channels," IEEE Trans. Commun., vol. 48, no. 3, pp. 401-415, Mar. 2000.

[2] Y. Ma and C. Chai, "Unified error probability analysis for generalized selection combining in Nakagami fading channels," IEEE J. Sel. Areas Commun., vol. 18, no. 11, pp. 2198-2210, Nov. 2000.

[3] M.-S. Alouini and M. K. Simon, "Performance of generalized selection combining over Weibull fading channels," Wireless Commun. Mob. Com., vol. 8, pp. 1077-1084, Jan. 2006.

[4] P. S. Bithas, G. K. Karagiannidis, N. C. Sagias, P. T. Mathiopoulos, S. A. Kotsopoulos, and G. E. Corazza, "Performance analysis of a class of GSC receivers over nonidentical Weibull fading channels," IEEE Trans. Veh. Technol., vol. 54, no. 6, pp. 1963-1970, Nov. 2005.

[5] M.-S. Alouini and M. K. Simon, "Performance of coherent receivers with hybrid SC/MRC over Nakagami- $m$ fading channels," IEEE Trans. Veh. Technol., vol. 48, no. 4, pp. 1155-1164, July 1999.

[6] V. A. Aalo, T. Piboongungon, and C.-D. Iskander, "Bit-error rate of binary digital modulation schemes in generalized Gamma fading channels," IEEE Commun. Lett., vol. 9, no. 2, pp. 139-141, Feb. 2005.

[7] N. C. Sagias and P. T. Mathiopoulos, "Switched diversity receivers over generalized Gamma fading channels," IEEE Commun. Lett., vol. 9, no. 10, pp. 871-873, Oct. 2005.

[8] M. D. Yacoub, "The $\alpha-\mu$ distribution: A physical fading model for the Stacy distribution," IEEE Trans. Veh. Technol., vol. 56, no. 1, pp. 27-34, Jan. 2007.

[9] I. S. Gradshteyn and I. M. Ryzhik, Table of Integrals, Series, and Products, 6th ed. New York: Academic Press, 2000.

[10] M. Abramowitz and I. A. Stegun, Handbook of Mathematical Functions, with Formulas, Graphs, and Mathematical Tables, 9th ed. New York: Dover, 1972. 